\title{
Magnetic resonance imaging-directed biopsy improves the prediction of prostate cancer aggressiveness compared with a 12-core transrectal ultrasound-guided prostate biopsy
}

\author{
JIE ZHANG ${ }^{1}$, JIANJUN XIU ${ }^{1}$, YIN DONG $^{1}$, MUWEN WANG $^{2}$, XUE HAN $^{1}$, YEJUN QIN ${ }^{3}$, \\ ZHAOQIN HUANG $^{1}$, SHIFENG CAI ${ }^{1}$, XIANSHUN YUAN $^{1}$ and QINGWEI LIU ${ }^{1}$ \\ ${ }^{1}$ Department of Radiology, ${ }^{2}$ Minimally Invasive Urology Center and ${ }^{3}$ Department of Pathology, \\ Provincial Hospital, Shandong University, Jinan 250021, P.R. China
}

Received October 22, 2013; Accepted February 17, 2014

DOI: $10.3892 / \mathrm{mmr} .2014 .1994$

\begin{abstract}
The Gleason grading system is a fundamental indicator of the aggressive nature of prostate cancer (PCa). Diffusion-weighted imaging (DWI) and magnetic resonance (MR) spectroscopy (MRS) are methods for the assessment of PCa aggressiveness. The present study was designed to prospectively investigate whether transrectal ultrasound (TRUS)-guided MR imaging (MRI)-directed biopsies (TRUS-MR-Dbs) improve the prediction of PCa aggressiveness in comparison with 12-core TRUS-guided biopsies (TRUS-Gbs). A total of 518 patients underwent pre-biopsy multi-parametric MRI to identify the clinically suspicious PCa regions. TRUS-MR-Dbs were performed on patients with suspected PCa by MRI in addition to TRUS-Gbs. Only patients who underwent radical prostatectomy (RP) were included in the comparative analysis. TRUS-biopsy was directed to those areas within suspicious regions with a minimum apparent diffusion coefficient obtained by DWI or with a maximum (choline + creatine)/citrate ratio obtained by MRS. The highest Gleason grades (HGGs) and the Gleason scores (GSs) of specimens were identified. The biopsies and RP results were evaluated using a McNemar test or $\chi^{2}$ analyses using Fisher' exact tests. MRI results were positive in $254(49.0 \%)$ of the 518 patients. TRUS-MR-Db detected 165/254 (65.0\%) cancer cases and TRUS-Gb detected 190/518 (36.7\%) cancer cases. Forty patients underwent RP. The TRUS-MR-Dbs method demonstrated a higher concordance rate (CR) with RP $(89.6 \%)$ than TRUS-Gbs (72.9\%, $\mathrm{P}=0.008$ ) for the overall HGG. The CRs with RP for
\end{abstract}

Correspondence to: Dr Qingwei Liu, Department of Radiology, Provincial Hospital, Shandong University, 324 Jingwu Road, Jinan 250021, P.R. China

E-mail: doctorlqw@163.com

Key words: prostate cancer, magnetic resonance imaging-directed biopsy, aggressiveness, Gleason grade, diffusion-weighted imaging, magnetic resonance spectroscopy
TRUS-MR-Dbs vs. those for TRUS-Gbs were 100 vs. $85.7 \%$ $(\mathrm{P}=0.5), 87.5$ vs. 68.8\% $(\mathrm{P}=0.031)$ and $50 \mathrm{vs.} 50 \%(\mathrm{P}=1)$ for HGG3, HGG4 and HGG5, respectively. The HGG CRs with RP for DWI-directed biopsies (DWI-Dbs) vs. MRS-directed biopsies (MRS-Dbs) were 77.1 vs. $50.0 \%(\mathrm{P}=0.015)$ for the overall tumors, 80.0 vs. $40.0 \%(\mathrm{P}=0.003)$ for peripheral zone tumors and 69.2 vs. $76.9 \%(\mathrm{P}=1)$ for transition zone tumors. A total of $37(77.1 \%)$ and $25(52.1 \% ; \mathrm{P}=0.007)$ tumors were assigned accurate GS for TRUS-MR-Dbs and TRUS-Gbs, respectively. The results revealed that TRUS-MR-Dbs improved the prediction of PCa aggressiveness and that DWI-Dbs had a superior performance when compared with MRS-Dbs in the peripheral zone.

\section{Introduction}

The precise determination of prostate cancer (PCa) aggressiveness facilitates the application of more personalized treatment regimens and improves the prediction of the prognosis for patients. The Gleason grade may be used to indicate the pathological characteristics of PCa. The Gleason score (GS) is a fundamental biological manifestation of the aggressiveness and prognosis of PCa $(1,2)$. The 12-core extended systematic transrectal ultrasound (TRUS)-guided biopsy (TRUS-Gb) is currently an accepted standard method to detect tumors and identify the tumors' Gleason grades. This contributed to the detection of low-volume, low-risk tumors, but the TRUS-Gb-determined GS is often increased in numerous patients receiving radical prostatectomy $(\mathrm{RP})(3,4)$.

Multi-parametric magnetic resonance imaging (MRI) methods, including T2-weighted imaging (T2WI), diffusion-weighted imaging (DWI), MR spectroscopy (MRS) and dynamic contrast-enhanced (DCE) imaging, are important in the accurate detection of PCa $(5,6)$. MRI-targeted biopsy is a potential alternative to improve the detection of PCa (7-10). DWI evaluates the random Brownian motion properties of water molecules in tissues, which are sensitive to water diffusion restriction. MRS is a non-invasive imaging modality to obtain metabolic information about tumors. DWI and MRS have demonstrated promising results in assessing $\mathrm{PCa}$ aggressiveness (11-13). 
Table I. MRI procedures and the corresponding parameters.

\begin{tabular}{lcccccc}
\hline Sequences & TR $(\mathrm{ms})$ & TE $(\mathrm{ms})$ & $\mathrm{ST}(\mathrm{mm})$ & Average & FOV $\left(\mathrm{cm}^{2}\right)$ & Matrix \\
\hline Axial T2WI (TSE) & 3110.00 & 101.00 & 3 & 2 & $20.0 \times 20.0$ & $320 \times 256$ \\
Coronal T2WI (TSE) & 2950.00 & 96.00 & 3 & 2 & $20.0 \times 20.0$ & $320 \times 256$ \\
Sagittal T2WI (TSE) & 3410.00 & 102.00 & 3 & 2 & $20.0 \times 20.0$ & $320 \times 256$ \\
MRS $^{\mathrm{a}}$ (CSI-PRESS) & 750.00 & 145.00 & - & 6 & - & - \\
DWI $^{\mathrm{b}}$ (EPI) & 6200.00 & 93.00 & 3 & 6 & $20.0 \times 20.0$ & $160 \times 120$ \\
Axial T1WI (TSE) $_{\text {Axial T2WI (TSE) }}^{467.00-645.00}$ & 4070.00 & 9.80 & 4 & 1 & $20.0 \times 20.0$ & $512 \times 384$ \\
Axial T1WI (VIBE) & 3.90 & 1.40 & 5 & 1 & $38.0 \times 28.5$ & $320 \times 168$ \\
Axial T1WI-DCE (VIBE) & 5.21 & 1.80 & 3 & 1 & $38.0 \times 30.8$ & $320 \times 182$ \\
\end{tabular}

aresolution was $7 \times 7 \times 7 \mathrm{~mm}^{3}$; b $b$-values $=0$ and $800 \mathrm{sec} / \mathrm{mm}^{2}$; c the sequence was performed prior to and following the injection of the contrast agent. TR, repetition time; TE, echo time; ST, slice thickness; FOV, field of view; TSE, turbo spin-echo sequence; EPI, single-shot echo-planar imaging; CSI-PRESS, 3D chemical shifting imaging techniques based on point-resolved spectroscopic sequence; DCE, dynamic contrast enhancement imaging; VIBE, volume interpolated body examination; MRI, magnetic resonance imaging; T1WI, T1-weighted imaging; T2WI, T2-weighted imaging; DWI, diffusion-weighted imaging; MRS, magnetic resonance spectroscopy.

The purpose of the present study was to investigate whether TRUS-guided MRI-directed biopsies (TRUS-MR-Dbs), including DWI-directed biopsies (DWI-Dbs) and MRS-directed biopsies (MRS-Dbs), may improve the prediction of $\mathrm{PCa}$ aggressiveness in patients when compared with the 12-core TRUS-Gbs.

\section{Materials and methods}

Ethics statement. The Ethical Committee of the Provincial Hospital Affiliated to Shandong University (Shandong, China) approved this prospective study and written informed consent was obtained from all of the participants.

Patients. The general practice of urologists in China and preference of the majority of patients is to undergo MRI examination prior to biopsy rather than following biopsy. As a routine examination, patients with clinically suspicious PCa (total prostate specific antigen (tPSA), $>4.0 \mathrm{ng} / \mathrm{ml}$; free PSA (fPSA)/tPSA, <0.16; positive findings from digital rectal examination) usually underwent a pre-biopsy MRI in the Radiology Department of Provincial Hospital (Shandong University, Shandong, China). From January 2010 to August 2013, 518 patients with clinically suspicious PCa were referred to undergo MRI examination by TRUS-Gbs. Patients with pretreatment, including hormone, radiation and surgery, had been excluded.

MRI. All MRI examinations were performed on a 3.0-T system (Magnetom verio; SIEMENS, Munich, Bavaria, Germany) with integrated eight-channel pelvic phased-array surface coils and spine coils for signal reception. All patients were imaged in a supine, head first position.

MR imaging sequences included T2WI,DWI, DCE imaging and MRS, among others. The parameters of sequences are summarized in Table I. In Table I, the sequences with a small field of view (FOV; 20x20 $\mathrm{cm}^{2}$ ) were used to observe PCa and the invasion of adjacent structures, including organ-confined tumors, extracapsular extension and seminal vesicle invasion. The sequences with large FOVs were used to observe nodal metastases and distant spread. Coronal and/or sagittal enhanced T1-weighted imaging as well as volume interpolated body examination with large FOVs were optional and are not listed in Table I. T2WI was performed with a turbo spin-echo sequence. In order to facilitate positioning, the center of slice groups, slice thickness and FOV used for DWI and DCE imaging were consistent with those of the axial T2WI with small FOVs. MRS data were overlaid on the corresponding axial T2W images. The MRS followed axial, coronal and sagittal T2WI to avoid the non-correspondence between the spectra and the corresponding voxel due to organ movement. DWI was performed using a single-shot echo-planar imaging technique. Apparent diffusion coefficient (ADC) maps were automatically generated from the DWI by the scanner. The MRS was performed using 3D chemical shifting imaging techniques based on a point-resolved spectroscopic sequence with sufficient lipid and water suppression. Eight saturation bands were used to minimize the contamination from adjacent structures of the prostate. Prior to approval for evaluation, a spectroscopist validated the spectra by examining them with regard to the correct positions, signal-to-noise ratio (SNR) $>5: 1$, full width at half maximum $(\mathrm{FWHM}) \leq 15 \mathrm{~Hz}$, a relatively steady baseline and the absence of lipid signals.

Two senior radiologists determined the PCa suspicious regions in consensus using the combined information of multi-parametric MRI. A lesion fulfilling two or more of the following criteria was regarded as a PCa suspicious region: i) An area with homogeneous low-signal-intensity and mass effect in the T2W images; ii) focal hyperintensity in diffusion-weighted images and corresponding hypointensity in ADC maps; iii) a normal choline (Cho) + creatine $(\mathrm{Cr}) /$ citrate (Cit) ratio $(\mathrm{CC} / \mathrm{C})=0.22 \pm 0.12$ in the peripheral zone $(\mathrm{PZ})$ and $(\mathrm{CC} / \mathrm{C})=0.34 \pm 0.14$ in the transition zone (TZ) (14) and $\mathrm{CC} / \mathrm{C}>0.34$ [the mean \pm standard deviation] in $\mathrm{PZ}$ and $\mathrm{CC} / \mathrm{C}>0.48$ in $\mathrm{TZ}$ and iv) a lesion that enhances rapidly and is washout of contrast agent in delay phase. 


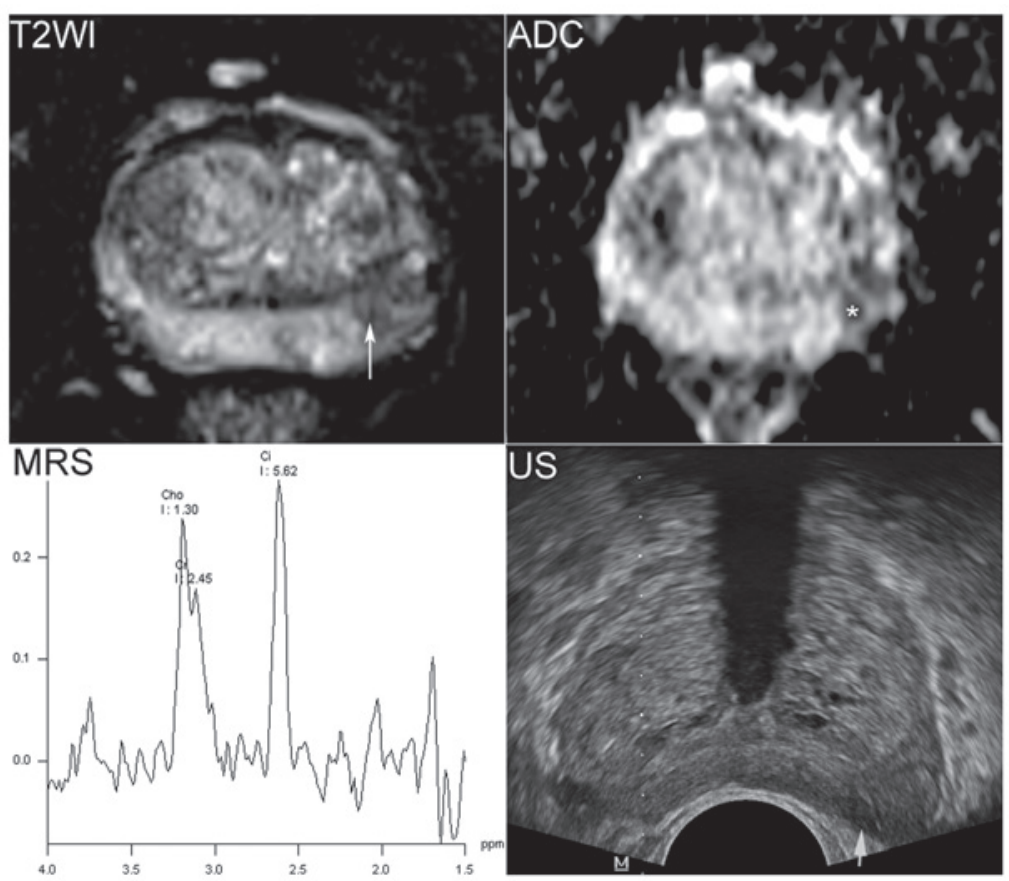

Figure 1. Transrectal ultrasound-guided magnetic resonance imaging-directed biopsy with the minimum ADC and maximum $(\mathrm{Cho}+\mathrm{Cr}) / \mathrm{Cit}(\mathrm{CC} / \mathrm{C})$ at the same site. $68 \mathrm{Y}, \mathrm{PSA}=6.12 \mathrm{ng} / \mathrm{ml}$. T2WI has a low-signal-intensity region (arrow). On the ADC map, diffusion restriction is visible at the same site with the minimum $\mathrm{ADC}$ at the site indicated by *. The corresponding MRS demonstrates $\mathrm{CC} / \mathrm{C}=0.67$. Transrectal US revealed a hypoechoic nodule. The white arrow points to the biopsy site. ADC, apparent diffusion coefficient; Cho, choline; Cr, creatine; Cit, citrate; MRS, magnetic resonance spectroscopy; T2WI, T2-weighted imaging; US, ultrasound.
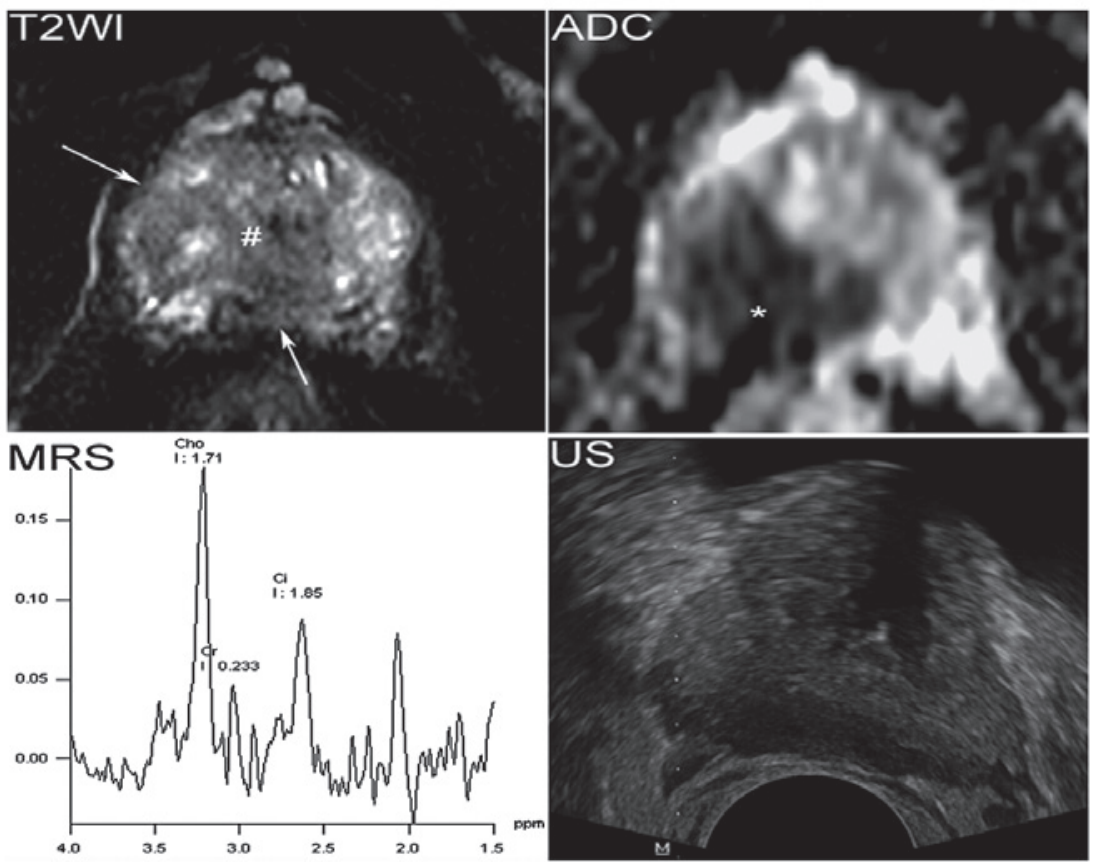

Figure 2. Transrectal ultrasound-guided magnetic resonance imaging-directed biopsy with the minimum ADC and maximum $(\mathrm{Cho}+\mathrm{Cr}) / \mathrm{Cit} \mathrm{CC} / \mathrm{C}$ at different sites; $75 \mathrm{Y}, \mathrm{PSA}=33.32 \mathrm{ng} / \mathrm{ml}$. T2WI has a large tumor in the right peripheral zone (arrows). The ADC map has a low-signal-intensity region with minimum $\mathrm{ADC}$ at the site indicated by *. The maximum CC/C value 1.05 obtained from MRS is indicated by " on the T2WI. Transrectal US revealed a hypoechoic tumor. ADC, apparent diffusion coefficient; Cho, choline; Cr, creatine; Cit, citrate; MRS, magnetic resonance spectroscopy; T2WI, T2-weighted imaging; US, ultrasound.

Biopsy and histopathological analysis. The median interval time from MRI examination to biopsy was three days (range, 1-12 days). Considering the areas with the minimum $\mathrm{ADC}$ or with the maximum $\mathrm{CC} / \mathrm{C}$ within suspicious regions may represent the most abnormal part with the highest cellularity or metabolism $(15,16)$. These areas were used to target the biopsies to represent the highest grade of the tumor (Figs. 1 and 2). The patients with suspected PCa on MR images under- 
went TRUS-MR-Dbs besides TRUS-Gbs (12-core) using an ultrasound device (DC-7; Mindray Medical International Limited, Shenzhen, China). The biopsy was performed by an urologist and a radiologist. The radiologist was also involved in the interpretation of the MR images. The steps of biopsy were as follows: In step 1, the 12-core systematic TRUS-Gbs were performed on all patients. In step 2, the area with minimum $\mathrm{ADC}$ in the suspicious regions was visually matched with the corresponding location identified by TRUS. One- to two-core biopsy was performed in this area while monitoring by TRUS. This step is known as DWI-Db. In step 3, the area with the maximum $\mathrm{CC} / \mathrm{C}$ in the suspicious regions was visually matched with the corresponding location identified using TRUS. One- to two-core biopsy was performed in this area while monitoring by TRUS. This step is known as MRS-Db. In step 4, one- to four-core biopsy was performed under the TRUS in the suspicious regions in MR images in addition to the biopsies performed in steps 2 and 3 .

If there were no suspicious regions of PCa on MRI, only step 1 was performed. If the minimum ADC and the maximum $\mathrm{CC} / \mathrm{C}$ appeared in the same site, steps 2 or 3 were omitted. The combination of steps $2-4$ is referred to as TRUS-MR-Db in the present study. The highest Gleason grade (HGG) for TRUS-MR-Db was obtained from step 2 and/or step 3. The Gleason score (GS) for TRUS-MR-Db was obtained from step 2, step 3 and step 4. The HGG and GS for TRUS-Gb were obtained from step 1.

TRUS was able to provide dynamic anatomy images of arbitrary sections. The majority of PCa cases were present as hypoechoic masses. However, the sonographic findings of PCa were non-specific. Numerous tumors were iso-echoic on TRUS. To achieve a good spatial consistency between ultrasound scans and MRI sections in performing TRUS-MR-Dbs (step 2, 3 and 4), certain criteria were required to be met for the biopsy: The gland morphology determined using ultrasound axial and sagittal scans being similar to that obtained with the axial and sagittal T2W images, in regard to specific anatomic structures (i.e. seminal vesicles, veromontanum, urethra and a number of hyperplasia-nodules) as landmarks. The biopsy cores were labeled to specify the location of the biopsy and marked on the $\mathrm{T} 2 \mathrm{~W}$ images at the corresponding sites.

Collectively, 12-18 cores were obtained from each patient. A median of four cores (range, two-six), which contained a median of two cores (range, one-three) in the most abnormal parts, were obtained from the suspicious regions for $\mathrm{PCa}$ in each patient.

The sites of suspicious PCa and their number were recorded on MRI for each patient. TRUS-MR-Dbs cores corresponding to each suspicious tumor on MRI were also recorded. The sites of 12 cores of TRUS-Gbs were relatively fixed. In the present study, only the patients who underwent RP were included to predict the aggressiveness of the PCa. The number of tumors was confirmed by RP pathology. For the patients who underwent RP, the urologist, the pathologist and the radiologist confirmed in consensus which cores of TRUS-Gbs and which cores of TRUS-MR-Dbs corresponded with each tumor using the biopsy records, the biopsy/RP pathology and the MR images as references. The biopsy cores corresponding to each tumor in a patient were then confirmed by the two biopsy methods. Only the tumors visible on MR images were included.
Table II. Clinical results of patients and tumors.

\begin{tabular}{lc}
\hline Parameters & Values \\
\hline Patients, $\mathrm{n}$ & 40 \\
Median age, years (range) & $66(55-78)$ \\
Mean tPSA, ng/ml (range) & $17.63(2.14-100)$ \\
Mean fPSA/tPSA x 100\% (range) & $13.63 \%(2.03-33.02 \%)$ \\
Stage & \\
T2 & 21 \\
T3 & 19
\end{tabular}

Gleason score

Peripheral zone

$3+2$

$3+3$

1

$3+4$

$4+3$

11

$4+4$ 14

$4+5$ 2

Transition zone

$2+3$

$3+2$

$3+3$

$3+4$

$4+3$

$4+4$

2

tPSA, total prostate specific antigen; fPSA, free prostate specific antigen.

The median interval time from biopsy to RP was nine days (2-33 days). Pathological sections of prostate from RP were matched with MR sections based on the level sextant locations by a pathologist and a radiologist. For cancerous biopsy cores and RP specimens, the GS of each tumor were determined by a pathologist with 15 years of experience, by the sum of the primary and secondary Gleason grades. The corresponding HGG of the specimens from the most aberrant regions was identified. The tumor volume was calculated by using the ellipsoid formula $(0.52 \mathrm{x}$ length $\mathrm{x}$ width $\mathrm{x}$ height).

Statistical analysis. Cross-tabulation analysis was used to describe the biopsy and RP findings (including HGG and GS). For both TRUS-Gbs and TRUS-MR-Dbs, the concordance rates (CRs) with RP were determined for HGG and GS. McNemar tests or $\chi^{2}$ analyses with Fisher's exact tests were performed to determine the differences for CRs with RP between TRUS-MR-Dbs and TRUS-Gbs as well as between DWI-Dbs and MRS-Dbs. The independent samples t-test was used to determine the difference between the volumes of tumors with accurate GS and of tumors with lower GS for TRUS-MR-Dbs. The correlation between tumor volume and HGG was evaluated using Pearson's correlation. For all statistical analyses, $\mathrm{P}<0.05$ was considered to indicate a statistically significant difference. Statistical analysis was performed with SPSS for Windows, version 17.0 (SPSS, Inc., Chicago, IL, USA). 
Table III. Cross tabulation for biopsies and radical prostatectomy results based on HGG grouping.

\begin{tabular}{lccccc}
\hline & & \multicolumn{3}{c}{ Radical prostatectomy result } & \\
\cline { 3 - 4 } Biopsy type & Biopsy result & HGG3 & HGG4 & HGG5 & PPV $(\%)$ \\
\hline TRUS-Gb & HGG0 & 2 & 0 & 0 & $0.0(0 / 2)$ \\
& HGG3 & 12 & 10 & 0 & $54.5(12 / 22)$ \\
& HGG4 & 0 & 22 & 1 & $95.7(22 / 23)$ \\
& HGG5 & 0 & 0 & 1 & $100.0(1 / 1)$ \\
TRUS-MR-Db & CR, \% (n) & $85.7(12 / 14)$ & $68.8(22 / 32)$ & $50.0(1 / 2)$ & $72.9(35 / 48)$ \\
& HGG3 & 14 & 4 & 0 & $77.8(14 / 18)$ \\
& HGG4 & 0 & 28 & 1 & $96.6(28 / 29)$ \\
& HGG5 & 0 & 0 & 1 & $100.0(1 / 1)$ \\
& CR, \% (n) & $100.0(14 / 14)$ & $87.5(28 / 32)$ & $50.0(1 / 2)$ & $89.6(43 / 48)$ \\
\hline
\end{tabular}

anot detection. HGG, highest Gleason grade; TRUS-Gb, 12-core transrectal ultrasound guided biopsy; TRUS-MR-Db, transrectal ultrasound-guided magnetic resonance imaging-directed biopsy; CR, concordance rate; PPV, positive predictive values.

Table IV. Comparation of concordance rates with radical prostatectomy between biopsies $(\mathrm{n}=48)$.

\begin{tabular}{lcr}
\hline For overall HGG & Percentage, \% (n) & P-value \\
\hline TRUS-MR-Db vs. TRUS-Gb & $89.6(43)$ vs. 72.9 (35) & 0.008 \\
DWI-Db vs. TRUS-Gb & $77.1(37)$ vs. 72.9 (35) & 0.727 \\
MRS-Db vs. TRUS-Gb & $50(24)$ vs. 72.9 (35) & 0.013 \\
DWI -Db vs. MRS-Db & $77.1(37)$ vs. 50 (24) & 0.015 \\
\hline
\end{tabular}

HGG, highest Gleason grade; TRUS-MR-Db, transrectal ultrasound-guided magnetic resonance imaging directed biopsy; TRUS-Gb, 12-core transrectal ultrasound-guided biopsy; DWI-Db, diffusion-weighted imaging directed biopsy; MRS-Db, magnetic resonance spectroscopy directed biopsy.

\section{Results}

Detection of cancer by TRUS-Gb. MRI results were positive in $254(49.0 \%)$ of the 518 patients. TRUS-MR-Db alone detected $165 / 254(65.0 \%)$ of cancer cases. TRUS-Gb alone detected $190 / 518(36.7 \%)$ of cancer cases. The overall number of patients with cancer was 196 . The median age of the 196 patients was 73 years (range, 51-87 years). The 165 cancer cases detected by TRUS-MR-Db included 159 of the 190 cancer cases, which were detected by TRUS-Gb, and six that were not able to be detected by TRUS-Gb. These six cancer cases had a HGG $\geq 3$. There were 31 cancer cases that were not detectable by TRUS-MR-Db, but by TRUS-Gb. Among these 31 cancer cases, 24 had a HGG of $\leq 3$ and 26 were $<0.5 \mathrm{~cm}$ in length.

$R P$ findings. Out of the 165 patients, only 40 patients underwent RP and the quality of MR images and MRS of these patients matched the criteria. The clinical results of 40 patients with 48 tumors (8 patients, 2 tumors/patient; 32 patients, 1 tumor/patient) are presented in Table II. Tables III and IV summarize the biopsy and RP findings of these patients. TRUS-MR-Db demonstrated a higher CR with RP for overall HGG, 89.6\% (43/48). For TRUS-Gb, it was $72.9 \%(35 / 48)$ $(\mathrm{P}=0.008)$. The CRs with RP for TRUS-MR-Dbs vs. those for TRUS-Gbs were $100 \%(14 / 14)$ vs. $85.7 \%(12 / 14 ; \mathrm{P}=0.5)$ for tumors with HGG of 3 (HGG3); $87.5 \%$ (28/32) vs. $68.8 \%$ $(22 / 32 ; \mathrm{P}=0.031)$ for tumors with HGG4 and $50 \%(1 / 2)$ vs. $50 \%(1 / 2 ; \mathrm{P}=1)$ for tumors with HGG5 (Fig. 3A). For biopsies with low grade (HGG3), the positive predictive value (PPV) for TRUS-MR-Dbs representing a true low grade was $77.8 \%$ $(14 / 18)$, whereas for TRUS-Gbs it was $54.5 \%(12 / 22 ; \mathrm{P}=0.125)$. Undergrading of tumors compared with RP was present at $10.4 \%$ (5/48) for TRUS-MR-Dbs and 27.1\% (13/48) for TRUS-Gb $(\mathrm{P}=0.008)$. Undergrading of tumors to $\mathrm{HGG}<4$, which had been graded as HGG4 or HGG5 by RP, occurred at $29.4 \%$ (10/34) for TRUS-Gbs and $11.8 \%$ (4/34) for TRUS-MR-Dbs $(\mathrm{P}=0.031)$. No overgrading was observed for the biopsies.

The HGG CRs with RP determined by DWI-Dbs vs. MRS-Dbs were $77.1 \%(37 / 48)$ vs. $50.0 \%(24 / 48 ; \mathrm{P}=0.015)$ for overall tumors; $80.0 \%(28 / 35)$ vs. $40.0 \%(14 / 35 ; \mathrm{P}=0.003)$ for PZ tumors and $69.2 \%(9 / 13)$ vs. $76.9 \%(10 / 13 ; \mathrm{P}=1)$ for TZ tumors. For DWI, the difference of HGG CRs with RP between $\mathrm{PZ}$ and TZ tumors was not significant $(\mathrm{P}=0.43)$. For MRS, the difference between PZ and TZ tumors was significant $(\mathrm{P}=0.023)$.

GS findings. A total of $37(77.1 \%)$ and $25(52.1 \% ; \mathrm{P}=0.004)$ tumors were assigned the same GS as with RP by 
Table V. Cross tabulation for biopsies and radical prostatectomy results based on GS grouping.

\begin{tabular}{|c|c|c|c|c|c|c|c|c|c|}
\hline \multirow[b]{2}{*}{ Biopsy type } & \multirow[b]{2}{*}{ Biopsy result } & \multicolumn{7}{|c|}{ Radical prostatectomy result } & \multirow[b]{2}{*}{ PPV, \% (n) } \\
\hline & & $2+3$ & $3+2$ & $3+3$ & $3+4$ & $4+3$ & $4+4$ & $4+5$ & \\
\hline \multirow[t]{9}{*}{ TRUS-Gb } & GS0 & 0 & 1 & 1 & 0 & 0 & 0 & 0 & $0.0(0 / 2)$ \\
\hline & $2+3$ & 1 & 1 & 0 & 0 & 0 & 0 & 0 & $50.0(1 / 2)$ \\
\hline & $3+2$ & 0 & 3 & 2 & 0 & 0 & 0 & 0 & $60.0(3 / 5)$ \\
\hline & $3+3$ & 0 & 0 & 5 & 6 & 4 & 0 & 0 & $33.3(5 / 15)$ \\
\hline & $3+4$ & 0 & 0 & 0 & 7 & 6 & 0 & 0 & $53.8(7 / 13)$ \\
\hline & $4+3$ & 0 & 0 & 0 & 0 & 6 & 1 & 0 & $85.7(6 / 7)$ \\
\hline & $4+4$ & 0 & 0 & 0 & 0 & 0 & 2 & 1 & $66.7(2 / 3)$ \\
\hline & $4+5$ & 0 & 0 & 0 & 0 & 0 & 0 & 1 & $100.0(1 / 1)$ \\
\hline & $\mathrm{CR}, \%(\mathrm{n})$ & $100(1 / 1)$ & $60(3 / 5)$ & $62.5(5 / 8)$ & $53.8(7 / 13)$ & $37.5(6 / 16)$ & $66.7(2 / 3)$ & $50(1 / 2)$ & $52.1(25 / 48)$ \\
\hline \multirow[t]{8}{*}{ TRUS-MR-Db } & $2+3$ & 1 & 1 & 0 & 0 & 0 & 0 & 0 & $50.0(1 / 2)$ \\
\hline & $3+2$ & 0 & 4 & 1 & 0 & 0 & 0 & 0 & $80.0(4 / 5)$ \\
\hline & $3+3$ & 0 & 0 & 7 & 2 & 2 & 0 & 0 & $63.6(7 / 11)$ \\
\hline & $3+4$ & 0 & 0 & 0 & 10 & 2 & 0 & 0 & $83.3(10 / 12)$ \\
\hline & $4+3$ & 0 & 0 & 0 & 1 & 11 & 0 & 0 & $91.7(11 / 12)$ \\
\hline & $4+4$ & 0 & 0 & 0 & 0 & 1 & 3 & 1 & $60.0(3 / 5)$ \\
\hline & $4+5$ & 0 & 0 & 0 & 0 & 0 & 0 & 1 & $100.0(1 / 1)$ \\
\hline & $\mathrm{CR}, \%(\mathrm{n})$ & $100(1 / 1)$ & $80(4 / 5)$ & $87.5(7 / 8)$ & $76.9(10 / 13)$ & $68.8(11 / 16)$ & $100(3 / 3)$ & $50(1 / 2)$ & $77.1(37 / 48)$ \\
\hline
\end{tabular}

GS, Gleason score; GS0, not detected; TRUS-Gb, 12-core transrectal ultrasound guided biopsy; TRUS-MR-Db, transrectal ultrasound-guided MR imaging-directed biopsy; CR, concordance rate; PPV, positive predictive values.

A

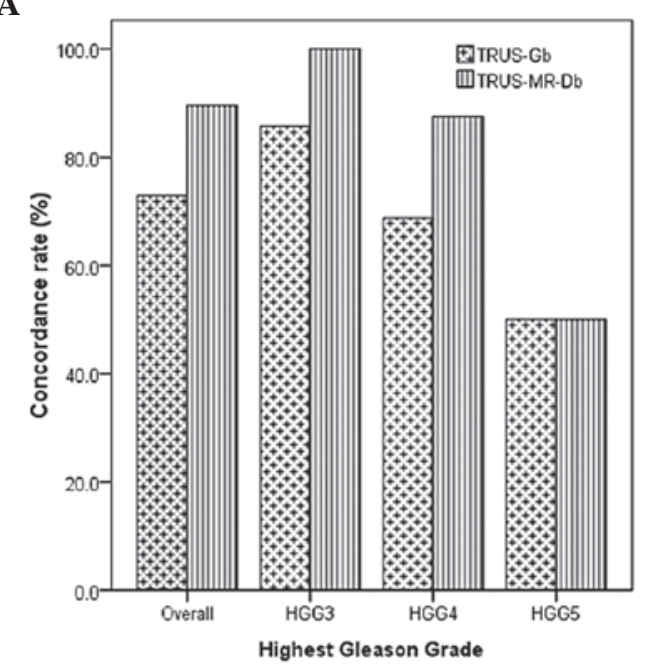

B

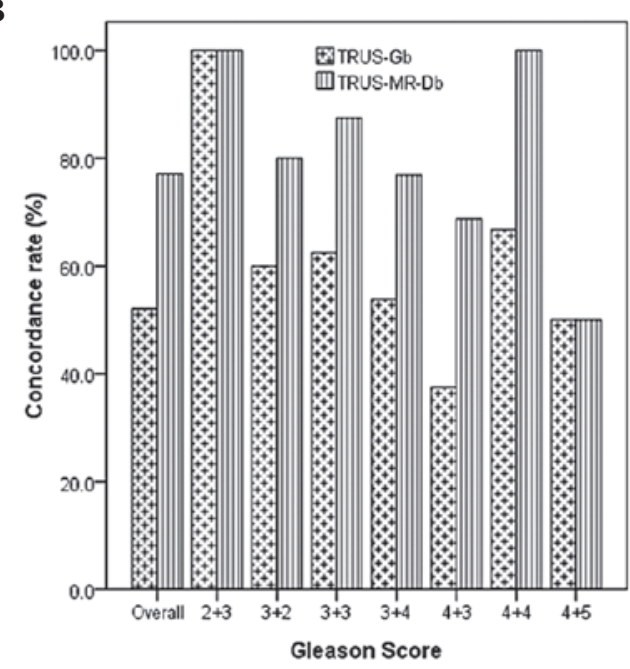

Figure 3. Concordance rates. (A) Concordance rates according to HGG categorization; 12-core TRUS-Gb vs. TRUS-MR-Db. (B) Concordance rates according to Gleason score categorization; 12-core TRUS-Gb vs. TRUS-MR-Db. HGG, highest Gleason grade; TRUS-Gb, 12-core transrectal ultrasound guided biopsy; TRUS-MR-Db, transrectal ultrasound-guided magnetic resonance imaging-directed biopsy.

TRUS-MR-Dbs and TRUS-Gbs, respectively (Fig. 3B). By TRUS-MR-Dbs and TRUS-Gbs, 9 (18.8\%) and 23 (47.9\%; $\mathrm{P}<0.001)$ tumors were assigned a lower GS than that assigned by RP, respectively. Two (4.5\%) tumors were assigned a higher GS than that assigned by RP for TRUS-MR-Dbs. The GSs determined by biopsies and RP are presented in Table V. The volume of tumors assigned the same GS as RP for TRUS-MR-Dbs $\left(5.3 \pm 4.0 \mathrm{~cm}^{3}\right)$ was significantly smaller than that of the tumors assigned lower GS $\left(9.1 \pm 5.3 \mathrm{~cm}^{3} ; \mathrm{P}=0.02\right)$. The tumor volume demonstrated a positive correlation with the HGG ( $\mathrm{r}=0.396 ; \mathrm{P}=0.005)$.

\section{Discussion}

In the present prospective study, both TRUS-MR-Dbs and TRUS-Gbs were performed on the same patient, and assessed 
in terms of how clinical factors for different patients (i.e, age, PSA, prostate weight) affect the biopsy Gleason grade CRs with RP (17). The most abnormal ADC or CC/C areas for $\mathrm{PCa}$ following multi-parametric imaging were used to target the biopsies. These areas were expected to have corresponding tumors with the highest grading $(12,18)$. Therefore, HGG CRs for biopsies with RP were mainly investigated in the present study. To the best of our knowledge, this may be the first prospective report on the use of the combination of DWI and MRS to direct TRUS-guided biopsy to obtain PCa specimens that may be more representative of the true RP Gleason grade.

Although TRUS-Gb increased the number of detected cancer cases, the majority or those that were not detected by TRUS-MR-Db exhibited an $\mathrm{HGG} \leq 3$ or a length $<0.5 \mathrm{~cm}$. TRUS-MR-Db used fewer biopsy cores (a median value of four cores) in a fewer number of patients (49\% of patients) and identified a lower number of low grade or microfocal tumors. These results were consistent with the findings of previous studies and reviews $(8,19,20)$, where it was considered that TRUS-MR-Db may avoid the unnecessary diagnosis of insignificant $\mathrm{PCa}$.

The Gleason grades as determined by TRUS-MR-Dbs demonstrated a higher CR with RP compared with TRUS-Gbs. Biopsy tends to underestimate tumor grades, which may result in the undertreatment of patients. For example, patients with high grades (HGG4 and HGG5) may be incorrectly receiving a treatment that is also used for patients with low grades $(\mathrm{HGG}<4)$. The rate of underestimation compared with RP for TRUS-MR-Db was significantly lower than that for TRUS-Gb $(\mathrm{P}=0.031)$. These results were consistent with results of previous retrospective studies on the ability of DWI and MRS to assess tumor aggressiveness and serve as biomarkers to improve pretreatment prediction of HGG $(12,13,21)$.

However, TRUS-MR-Gbs only improved the prediction rates for tumors with HGG4 but not for tumors with HGG3 and 5. For tumors with HGG3, the GS of tumors visible on MR images was $3+3$, followed by $3+2$ in the present study, which demonstrated that the structure/grade of low-grade tumors was relatively homogeneous or mainly of grade 3 . Furthermore, the results of the present study revealed that a small tumor volume was associated with a low grade. Therefore, as long as the tumor was detected by 12-core TRUS-Gbs, the probability of detection of a component with grade 3 may be greater. San Francisco et al (22) have reported that tumors with low Gleason grade detected by extended biopsies ( $\geq 10$ cores) had significantly higher concordance $(88 \%)$ with the prostatectomy Gleason grade. In addition, the 12-core biopsy was performed under ultrasound guidance in the present study. The intensity of the relatively small low-grade tumors visible on MR images was relatively homogeneous, which may correspond to a homogeneous structure/grade in the low-grade tumors in the present study. For HGG5, the number of tumors was markedly low (2), which may be not sufficient for a reliable analysis. In addition, the intensity of high grade tumors was often heterogeneous, which may be associated with tumor necrosis. In addition, high grade tumors were often large, which increased the difficulty of the spatial consistency between MR images and ultrasound scans. Therefore, TRUS-MR-Dbs did not improve the prediction rate for HGG5.
DWI-Dbs performance was significantly superior compared with MRS-Dbs in assessing the HGG of PCa $(\mathrm{P}=0.015)$. TRUS-guided DWI-Dbs and MRS-Dbs improved the accuracy in the detection of PCa $(19,23,24)$. However, DWI-Db and TRUS-Gb CRs with RP for HGGs were not significantly different $(\mathrm{P}=0.727)$. CRs with RP for MRS-Dbs were lower than for TRUS-Gb ( $\mathrm{P}=0.013$; Table IV). The negative correlation between $\mathrm{ADC}$ and Gleason grade and the positive correlation between $\mathrm{CC} / \mathrm{C}$ and Gleason grade have been reported in previous studies $(13,18,25)$. Despite this, it is possible that the minimum $\mathrm{ADC}$ or maximum $\mathrm{CC} / \mathrm{C}$ of a tumor may not fully represent the highest grade of a tumor, particularly for $\mathrm{CC} / \mathrm{C}$. For only $50 \%$ of tumors, the HGGs were accurately predicted with the maximum $\mathrm{CC} / \mathrm{C}$. However, TRUS-MR-Dbs (the combination of DWI-Dbs and MRS-Dbs) may improve the CRs with RP compared with TRUS-Gbs. A previous prospective study reported that DWI targeted MR-guided biopsy significantly improved the pretreatment assessment of PCa aggressiveness compared with 10-core TRUS-guided biopsy (26). The possible reasons for the different results are as follows: (i) The location accuracy for TRUS-MR-Db is lower than that for MR-guided biopsy and (ii) the 12-core biopsy further improves the CRs with RP compared with 10-core biopsy (22). Despite this, the combination of DWI- and MRS-Dbs had an improved performance as compared with TRUS-Gb. DWI-Db has a superior performance to MRS-Db in predicting HGG for $\mathrm{PZ}$ tumors $(\mathrm{P}=0.003)$, but was equal to MRS-Db for TZ tumors $(\mathrm{P}=1)$. It may be associated with relatively strict requirements for MRS, including uniformity of a static magnetic field and stability of radiofrequency. Although eight saturation bands were used to minimize the contamination from adjacent structures of the prostate, a number of artifacts may have remained. The $\mathrm{PZ}$ adjacent to the rectum and pelvic fat is more susceptible to rectum gas, rectum peristalsis and lipid contamination. Voxels with poor quality MRS or artifacts were excluded. The majority of the excluded voxels were in PZ tumors. A number of the excluded voxels may have had the highest grade and certain included voxels may have contained hidden artifacts. Although DWI may also be affected by these artifacts, the impact on DWI was far less than that on MRS. A number of the artifacts of DWI may have been corrected or compensated in a better way. A retrospective study by Kobus et al (11) also reported that DWI assesses the PCa aggressiveness better in PZ.

The GS of PCa has a dominant role in the evaluation of tumor aggressiveness. The GS CRs with RP determined by TRUS-MR-Dbs and TRUS-Gbs were also compared. The GS determined by TRUS-MR-Dbs demonstrated a higher CR $(\mathrm{P}=0.004)$ and a lower underestimated rate $(\mathrm{P}<0.001)$ with $\mathrm{RP}$ than that determined by TRUS-Gbs; however, TRUS-MR-Dbs overestimated two tumors. The mean volume of tumors assigned accurate GS was smaller than that of underestimated tumors.

There were severallimitations in the present study, including the relatively low number of tumors examined, particularly the number of tumors with HGG5 (only two), which may reduce the validity of the results. In China, the incidence of PCa is lower than in Western countries. Although the incidence of PCa has significantly increased in recent years, the PSA 
screening is not commonly applied and the majority of patients are identified as having high grade PCa when diagnosed (27) and have lost the opportunity for RP. In China, patients with $\mathrm{PCa}$ are relatively old (median age $>70$ years) and therefore, patients tend to select a therapy associated with reduced injury, such as endocrine therapy instead of RP. Almost all patients with HGG5 had lost the opportunity of RP in the Provincial Hospital (Shandong, China), which may be the reason for the low number of HGG5 tumors. The CRs with RP of each GS group of biopsies were calculated, but statistical comparisons for the corresponding GS groups between TRUS-MR-Dbs and TRUS-Gbs were not performed due to the low number of each GS group tumors.

A second limitation was the spatial consistency between ultrasound scans and MRI sections. Although measures were taken to solve this problem, it was not easy to achieve the exact spatial consistency. An MRI/ultrasound fusion platform may improve the detection of tumors due to its relatively accurate spatial consistency $(19,28,29)$. To the best of our knowledge, this platform has not been used to predict tumor aggressiveness thus far. MRI-guided biopsy may locate the tumor more accurately and has been used to improve the prediction of $\mathrm{PCa}$ aggressiveness (26). However performing target biopsy under MRI-guidance is expensive, time-consuming and not widely available. TRUS-guided biopsies with the visual facilitations of MRI (DWI or MRS in the present study) have been prospectively demonstrated to contribute to the detection of $\mathrm{PCa}(7,24)$, and is currently the most widely used diagnostic strategy. The present study used the same biopsy method (TRUS-MR-Db) to detect the most abnormal part of PCa. Although the spatial consistency with TRUS-MR-Db between the ultrasound scans and MRI sections may be poorer than with the MRI/ultrasound fusion platform, TRUS-MR-Db was satisfactory to a certain extent. Therefore, TRUS-MR-Dbs (combination of DWI- and MRS-Dbs) demonstrated higher HGG and GS CRs for overall tumors with RP compared with TRUS-Gbs.

In conclusion, DWI- and MRS-directed biopsies at the most abnormal (lowest ADC or highest $\mathrm{CC} / \mathrm{C}$ ) sites may be necessary in the pretreatment and prediction of tumor aggressiveness in PCa. DWI-Dbs are more effective than MRS-Dbs, particularly for tumors in the PZ.

\section{Acknowledgements}

The present study was supported by the Shandong Province Science and Technology Development Plan (nos. 2012GSF11820 and 2012YD18053). The authors are grateful to the language services agent, Beijing chunfenglv, for grammatical support.

\section{References}

1. Gleason DF and Mellinger GT: Prediction of prognosis for prostatic adenocarcinoma by combined histological grading and clinical staging. J Urol 111: 58-64, 1974.

2. Egevad L, Granfors T, Karlberg L, Bergh A and Stattin P Prognostic value of the Gleason score in prostate cancer. BJU Int 89: 538-542, 2002.

3. Yang CW, Lin TP, Huang YH, et al: Does extended prostate needle biopsy improve the concordance of Gleason scores between biopsy and prostatectomy in the Taiwanese population? J Chin Med Assoc 75: 97-101, 2012.
4. Divrik RT, Eroglu A, Sahin A, Zorlu F and Ozen H: Increasing the number of biopsies increases the concordance of Gleason scores of needle biopsies and prostatectomy specimens. Urol Oncol 25: 376-382, 2007.

5. Delongchamps NB, Rouanne M, Flam T, et al: Multiparametric magnetic resonance imaging for the detection and localization of prostate cancer: combination of $\mathrm{T} 2$-weighted, dynamic contrast-enhanced and diffusion-weighted imaging. BJU Int 107: 1411-1418, 2011.

6. Mazaheri Y, Shukla-Dave A, Hricak H, et al: Prostate cancer: identification with combined diffusion-weighted MR imaging and 3D ${ }^{1} \mathrm{H}$ MR spectroscopic imaging - correlation with pathologic findings. Radiology 246: 480-488, 2008.

7. Park BK, Park JW, Park SY, et al: Prospective evaluation of 3-T MRI performed before initial transrectal ultrasound-guided prostate biopsy in patients with high prostate-specific antigen and no previous biopsy. AJR Am J Roentgenol 197: W876-W881, 2011.

8. Haffner J, Lemaitre L, Puech P, et al: Role of magnetic resonance imaging before initial biopsy: comparison of magnetic resonance imaging-targeted and systematic biopsy for significant prostate cancer detection. BJU Int 108: E171-E178, 2011.

9. Pinto PA, Chung PH, Rastinehad AR, et al: Magnetic resonance imaging/ultrasound fusion guided prostate biopsy improves cancer detection following transrectal ultrasound biopsy and correlates with multiparametric magnetic resonance imaging. J Urol 186: 1281-1285, 2011.

10. Vourganti S, Rastinehad A, Yerram NK, et al: Multiparametric magnetic resonance imaging and ultrasound fusion biopsy detect prostate cancer in patients with prior negative transrectal ultrasound biopsies. J Urol 188: 2152-2157, 2012.

11. Kobus T, Vos PC,Hambrock T,etal:Prostate cancer aggressiveness: in vivo assessment of MR spectroscopy and diffusion-weighted imaging at 3 T. Radiology 265: 457-467, 2012.

12. Hambrock T, Somford DM, Huisman HJ, et al: Relationship between apparent diffusion coefficients at 3.0-T MR imaging and Gleason grade in peripheral zone prostate cancer. Radiology 259: 453-461, 2011.

13. Kobus T, Hambrock T, Hulsbergen-van de Kaa CA, et al: In vivo assessment of prostate cancer aggressiveness using magnetic resonance spectroscopic imaging at $3 \mathrm{~T}$ with an endorectal coil. Eur Urol 60: 1074-1080, 2011.

14. Scheenen TW, Heijmink SW, Roell SA, et al: Three-dimensional proton MR spectroscopy of human prostate at $3 \mathrm{~T}$ without endorectal coil: feasibility. Radiology 245: 507-516, 2007.

15. Wang XZ, Wang B, Gao ZQ, et al: Diffusion-weighted imaging of prostate cancer: correlation between apparent diffusion coefficient values and tumor proliferation. J Magn Reson Imaging 29: 1360-1366, 2009.

16. Wang XZ, Wang B, Gao ZQ, et al: ${ }^{1} \mathrm{H}-\mathrm{MRSI}$ of prostate cancer: the relationship between metabolite ratio and tumor proliferation. Eur J Radiol 73: 345-351, 2010.

17. Stackhouse DA, Sun L, Schroeck FR, et al: Factors predicting prostatic biopsy Gleason sum under grading. J Urol 182: 118-124, 2009.

18. Zakian KL, Sircar K, Hricak H, et al: Correlation of proton MR spectroscopic imaging with gleason score based on step-section pathologic analysis after radical prostatectomy. Radiology 234: 804-814, 2005.

19. Delongchamps NB, Peyromaure M, Schull A, et al: Prebiopsy magnetic resonance imaging and prostate cancer detection: comparison of random and targeted biopsies. J Urol 189: 493-499, 2013.

20. Moore CM, Robertson NL, Arsanious N, et al: Image-guided prostate biopsy using magnetic resonance imaging-derived targets: a systematic review. Eur Urol 63: 125-140, 2013.

21. Verma S, Rajesh A, Morales H, et al: Assessment of aggressiveness of prostate cancer: correlation of apparent diffusion coefficient with histologic grade after radical prostatectomy. AJR Am J Roentgenol 196: 374-381, 2011.

22. San Francisco IF, DeWolf WC, Rosen S, Upton M and Olumi AF: Extended prostate needle biopsy improves concordance of Gleason grading between prostate needle biopsy and radical prostatectomy. J Urol 169: 136-140, 2003.

23. Park BK, Lee HM, Kim CK, Choi HY and Park JW: Lesion localization in patients with a previous negative transrectal ultrasound biopsy and persistently elevated prostate specific antigen level using diffusion-weighted imaging at three Tesla before rebiopsy. Invest Radiol 43: 789-793, 2008. 
24. Prando A, Kurhanewicz J, Borges AP, Oliveira EM Jr and Figueiredo E: Prostatic biopsy directed with endorectal MR spectroscopic imaging findings in patients with elevated prostate specific antigen levels and prior negative biopsy findings: early experience. Radiology 236: 903-910, 2005.

25. Tamada T, Sone T, Jo Y, et al: Apparent diffusion coefficient values in peripheral and transition zones of the prostate: comparison between normal and malignant prostatic tissues and correlation with histologic grade. J Magn Reson Imaging 28: 720-726, 2008.

26. Hambrock T, Hoeks C, Hulsbergen-van de Kaa C, et al: Prospective assessment of prostate cancer aggressiveness using 3-T diffusion-weighted magnetic resonance imaging-guided biopsies versus a systematic 10-core transrectal ultrasound prostate biopsy cohort. Eur Urol 61: 177-184, 2012.
27. Na R, Jiang H, Kim ST, et al: Outcomes and treands of prostate biopsy for prostate cancer in Chinese men from 2003 to 2011. PLoS One 7: e49914, 2012.

28. Miyagawa T, Ishikawa S, Kimura T, et al: Real-time Virtual Sonography for navigation during targeted prostate biopsy using magnetic resonance imaging data. Int J Urol 17: 855-860, 2010.

29. Rastinehad AR, Baccala AA Jr, Chung PH, et al: D'Amico risk stratification correlates with degree of suspicion of prostate cancer on multiparametric magnetic resonance imaging. J Urol 185: 815-820, 2011. 